Article

\title{
Wind Direction Inversion from Narrow-Beam HF Radar Backscatter Signals in Low and High Wind Conditions at Different Radar Frequencies
}

\author{
Wei Shen ${ }^{1,2, *}$ and Klaus-Werner Gurgel ${ }^{3}$ \\ 1 Key Laboratory of Intelligent Perception and Systems for High-Dimensional Information of Ministry of \\ Education, Nanjing University of Science and Technology, Nanjing 210094, Jiangsu, China \\ 2 School of Computer Science and Engineering, Nanjing University of Science and Technology, \\ Nanjing 210094, Jiangsu, China \\ 3 Institute of Oceanography, Faculty of Mathematics, Informatics and Natural Science, \\ University of Hamburg, D-20146 Hamburg, Germany; klaus-werner.gurgel@uni-hamburg.de \\ * Correspondence: weishen@njust.edu.cn; Tel.: +86-188-516-98456
}

Received: 31 July 2018; Accepted: 12 September 2018; Published: 16 September 2018

\begin{abstract}
Land-based, high-frequency (HF) surface wave radar has the unique capability of monitoring coastal surface parameters, such as current, waves, and wind, up to $200 \mathrm{~km}$ off the coast. The Doppler spectrum of the backscattered radar signal is characterized by two strong peaks that are caused by the Bragg-resonant scattering from the ocean surface. The wavelength of Bragg resonant waves is exactly half the radio wavelength (grazing incidence), and these waves are located at the higher frequency part of the wave spectral distribution. When HF radar operates at higher frequencies, the resonant waves are relatively shorter waves, which are more sensitive to a change in wind direction, and they rapidly respond to local wind excitation and a change in wind direction. When the radar operates at lower frequencies, the corresponding resonant waves are relatively longer and take longer time to respond to a change in wind direction due to the progress of wave growth from short waves to long waves. For the wind inversion from HF radar backscatter signals, the accuracy of wind measurement is also relevant to radar frequency. In this paper, a pattern-fitting method for extracting wind direction by estimating the wave spreading parameter is presented, and a comparison of the pattern-fitting method and a conventional method is given as well, which concludes that the pattern-fitting method presents better results than the conventional method. In order to analyze the wind direction inversion from radar backscatter signals under different wind conditions and at different radar frequencies, two radar experiments accomplished in Norway and Italy are introduced, and the results of wind direction inversion are presented. In the two experiments, the radar worked at 27.68 $\mathrm{MHz}$ and $12 \mathrm{MHz}$, respectively, and the wind conditions at the sea surface were quite different. In the experiment in Norway, $67.4 \%$ of the wind records were higher than $5 \mathrm{~m} / \mathrm{s}$, while, in the experiment in Italy, only $18.9 \%$ of the wind records were higher than $5 \mathrm{~m} / \mathrm{s}$. All these factors affect the accuracy of wind direction inversion. The paper analyzes the radar data and draws a conclusion on the influencing factor of wind direction inversion.
\end{abstract}

Keywords: wind direction; Bragg scattering; wave directional spreading pattern; HF radar

\section{Introduction}

The use of high-frequency $(\mathrm{HF})$ radar systems to measure ocean surface wind has been investigated for many years. The radar transmits high-frequency (3-30 MHz) radio waves that are scattered from ocean waves in all directions, with some scattered energy toward the radar's receiver. It provides the possibility of making synoptic measurements of ocean surface dynamics, such as currents, waves, 
and winds, over a coastal region of interest. Crombie [1] discovered that waves that are half the wavelength of the incident radio wavelength are represented by two dominant peaks on the Doppler spectrum. An example of a typical HF radar Doppler spectrum is illustrated in Figure 1.

Extraction of the wind direction from HF radar backscatter has been investigated for four decades. Long and Trizna [2] suggested using the relative amplitude of the two first-order peaks to determine the short wind wave, and hence, wind direction, and developed a simple model for this. They pointed out that the method has a left-right ambiguity relative to the radio beam, which has to be resolved using additional information. Stewart and Barnum [3] suggested using a half-cosine 2s-power directional model, $\cos ^{2 s}(\theta / 2)$, in the case of the assumption that there is no energy propagating in a direction against the wind, whereas it is always possible to observe both first-order Bragg peaks even when the wind is blowing along the radar beam. Tyler et al. [4] added a minor constant to the half-cosine function, and Zeng et al. [5] presented a modified method based on Tyler's work using broad-beam radar data. The hyperbolic secant function $0.5 \beta \operatorname{sech}^{2}(\beta \theta)$ model was found to provide a better agreement than the half-cosine type [6]. In Gurgel's work [7], the hyperbolic secant function that gives the most realistic up/down wind ratio conditions for 5-20-m-long ocean waves was identified. Heron [8,9] and Huang et al. [10] presented excellent work on the factors to be considered in developing algorithms for the extraction of wind direction using one radar station. Chu et al. [11] applied the multi-beam method for wind direction inversion from a hybrid skywave radar. It is clear from References $[12,13]$ that the spreading parameter used in describing the directional distribution of the ocean waves may have a critical influence on determining the wind directions. Kirincich $[14,15]$ and Shen et al. [12] recently illustrated that in situ wind data can be used to calibrate the wind inversion. A neural network method $[12,16]$ was also used for wind field inversion. For the research of radar measurement from ocean surface at different radar frequencies and sea states, Li et al. [17] presented some work on the wave measurements at low-frequency radar under low sea state. Halverson et al. [18] collected HF radar data over one year, containing different sea states, tides, and so on. During the experiment, the radar operated at a single frequency of $25 \mathrm{MHz}$, a relatively high frequency in the HF band.

The chief uncertainty of wind direction inversion lies in the dependence of most of the assumed wave directional distributions on wind speed, implying a dependence of the measured mean wind direction on some prior knowledge of wind speed $U_{10}$ [19], which impacts as a form of wave age $\left(c_{\mathrm{p}} / U_{10}\right)$. However, Hasselmann et al. [20] noted that the spreading function is more sensitive to $\left(f / f_{\mathrm{p}}\right)$ than to $\left(c_{\mathrm{p}} / U_{10}\right)$. In HF radar remote sensing, the value of $U_{10}$ and wave peak $f_{\mathrm{p}}$ are unknown, which makes it difficult to determine the spreading parameter. However, with two radar sites, the ambiguity of wind direction estimation could be eliminated and the directional spreading pattern could be fitted by the ratio of the first-order peak from two radar sites. Thus, this paper proposes a pattern-fitting method for determining the directional spreading parameter, as well as the wind direction.

Several kinds of HF radar systems were developed for ocean surface remote sensing. The Seasonde and OSMAR-S (ocean state measuring and analyzing radar, short ranges) series use the monopole and cross-loop as the receiving antenna, which is a kind of compact radar system that is easy to deploy. Some other radar systems use a linear array to form a narrow beam to achieve high azimuth resolution, such as the WERA system developed in Germany, using a 16-element monopole for the receiving antenna. During the experiment, a certain number of grids is defined (normally $n \times m$ ). With a linear array, a narrow beam can be formed to steer to the grid points on the radar maps. In this paper, the wind direction inversion is based on WERA radar experiments. 


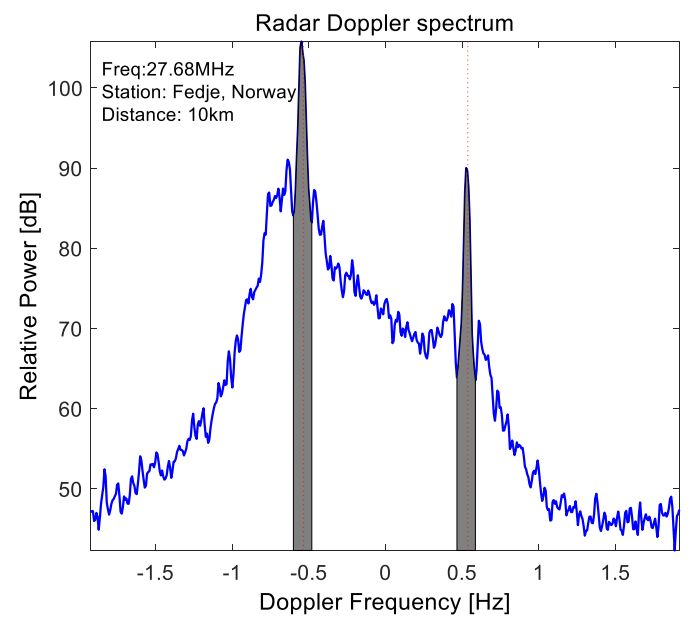

Figure 1. An example of the Doppler spectrum of a WavE RAdar (WERA) experiment in EuroROSE (European radar ocean sensing).

\section{Materials and Methods}

The ocean surface wind determines the directional spreading distribution of ocean waves. During the radar experiment, once a certain grid point (a small patch at the sea surface) is selected, the radio beam direction $\phi$ is given. The wind direction and the directional spreading pattern of resonant waves determine the strength of wave components receding from and approaching the radar site. Theoretical treatments by Barrick [21] and Robson [22] gave the first-order backscatter cross-section as follows:

$$
\sigma_{1}(\omega)=2^{6} \pi k_{0}^{4} \sum_{m= \pm 1} S\left(-2 m k_{0}\right) \delta\left(\omega-m \omega_{B}-2 k_{0} \cdot v\right)
$$

where $k_{0}$ is the Bragg wave number, $\omega_{B}$ is the angular Bragg frequency, and $S(\cdot)$ is the ocean wave spectrum. Equation (1) can be applied to evaluate the surface current velocity $v$ by observing the first-order frequency offset. The spectral energy density for the first-order backscattered radiation is proportional to the scattering cross section. Given that $\phi$ is the azimuth of the radio beam and $\theta$ is the wind direction, the power ratio of the first-order peaks is

$$
R(\theta)=\frac{\sigma_{1}\left(f_{B}\right)}{\sigma_{2}\left(f_{B}\right)}=\frac{G(\pi+\phi-\theta)}{G(\phi-\theta)},
$$

where $G(\theta)$ is the wave directional spreading function of Bragg waves, and it is made dimensionless and is normalized as follows:

$$
\int_{-\pi}^{\pi} G(f, \theta) d \theta=1
$$

\subsection{Power Ratio of a Radar's First-Order Peaks and Wave Directional Distribution}

There are many proposals for the form of the directional spreading function, such as cosine 2l-power-type spreading [23], circular-normal spreading [24], half-cosine 2s-power spreading [25], and hyperbolic secant-squared spreading [6]. In this paper, two widely used functions, namely the half-cosine 2s-power type and the hyperbolic secant-squared type, are discussed.

The basic form of the cosine-type spreading function was proposed by Longuet-Higgin et al. [25], and Mitsuyasu et al. [26] gave its form as follows:

$$
G(f, \theta)=\frac{2^{2 s-1}}{\pi} \frac{\Gamma^{2}(s+1)}{\Gamma(2 s+1)} \cdot \cos ^{2 s}\left(\frac{\theta}{2}\right),
$$


where $\Gamma$ denotes the Gamma-function and $s$ is the spreading parameter. Mitsuyasu et al. presented a reasonable comprehensive set of estimates for $s$ using measurements obtained from a cloverleaf buoy. Based on the argument that nonlinear processes primarily determine the wave spectrum, the spreading parameter $s$ might be expected to depend both on $U_{10} / C_{\mathrm{p}}$ and $f / f_{\mathrm{p}}$.

M. A. Donelan et al. proposed a hyperbolic secant function for the directional spectrum of wind-generated waves in deep water. The angular spreading is given as

$$
G(f, \theta)=0.5 \beta \operatorname{sech}^{2}(\beta \cdot \theta),
$$

where $\beta$ is the directional spreading parameter. The three-dimensional evolution of freely propagating, second-order Stokes gravity wave groups indicates that an envelope soliton group propagating around the main wave direction is described by a hyperbolic secant.

The main difference between the half-cosine 2s-power function and the hyperbolic secant function is that $G_{\cos }\left(\theta_{0}\right)=0$, while $G_{\text {sech }}\left(\theta_{0}\right)>0\left(\theta_{0}=\pi\right.$ or $\left.\theta_{0}=-\pi\right)$. That is to say, in the form of the hyperbolic secant-squared function, there is some wave energy transferring opposite to the wind direction. In an HF radar measurement, if the wind blows along the radio beam direction $\phi$, the measured approaching wave component (which is proportional to the positive first-order peak power) is not equal to zero. Even in the buoy measurement accomplished by M. A. Donelan et al. [6], there are some minor wave components propagating against the wind direction. Another difference is that the half-cosine function is periodic while the hyperbolic secant function is not. If the hyperbolic secant function is implemented in the wave direction spectrum while calculating the ratio of approaching and receding wave components, the direction should be separated into two conditions: $\theta \in[-\pi, 0]$ and $\theta \in[0, \pi]$.

\subsection{Wind Direction Inversion from a Radar's First-Order Backscatter}

As introduced in Equation (2), the power ratio of the Bragg peaks is a function of the wind-wave directional pattern $G(\theta)$ and the wind direction $\phi$; thus, the wind direction can be written as

$$
\theta_{i}=\phi_{i} \pm \Theta\left(R_{i}\right),(i=1,2)
$$

where $\Theta\left(R_{i}\right)$ is the angle between the radio beam direction and the wind direction, which is derived from the power ratio of the first-order peaks combined with the wave directional distribution function. In order to determine the wind direction from the radar Doppler spectrum, the ambiguity, which consists of two possible choices, in Equation (6) should be removed. Attempts using a variety of methods were made, including (1) general wind circulation information from maps of air pressure; (2) two radar seats in different locations, which help find the wind direction estimation with the smallest error [7]; and (3) one radar switching between radio beam directions [10,27].

Gurgel [7] proposed a unique solution for direction determination by means of the least-square minimum (LSM) principle:

$$
\left[R_{1}-\frac{G\left(\phi_{1}-\theta_{k}+\pi\right)}{G\left(\phi_{1}-\theta_{k}\right)}\right]^{2}+\left[R_{2}-\frac{G\left(\phi_{2}-\theta_{k}+\pi\right)}{G\left(\phi_{2}-\theta_{k}\right)}\right]^{2}=\min ,
$$

where $R_{1}$ and $R_{2}$ are the ratio of the Bragg peak power, and $\phi_{1}$ and $\phi_{2}$ are radio beam directions. Once a grid point is selected, the radio beam directions are determined. $G(\cdot)$ is the directional spreading function $\cos ^{2 \mathrm{~s}}(\theta / 2)$, for which the values of spreading parameter $s$ were suggested as $s=1$ or $s=2$; $\theta_{k}$ is the variable of the LSM, which is the direction of the wind wave. In the estimation of LSM, $\theta_{k} \in\left[0 \sim 360^{\circ}\right]$. Finally, one value of $\theta_{k}$ is found that gives the minimum of Equation (7). In Gurgel's work [7], he also suggested using $\operatorname{sech}^{2}(\beta \cdot \theta)$ with a spreading value of $\beta=0.8$, which is based on the statistical Bragg peak ratio range. All these direction patterns have the limitation that the spreading parameter is fixed to a constant value. 
The chief uncertainty of the traditional methods lies in the dependence of most of the assumed wave directional distributions on wind speed (with a constant value for the spreading parameter $s$ or $\beta$, except for the method of M. Heron [8]). The spreading distribution implies the dependence of the measured mean wind direction on some prior knowledge of wind speed [19]. The multi-beam method proposed by $\mathrm{M}$. Heron also assumes that the wind direction $\theta$ in all cells is identical, which might not be the case in reality.

\subsection{Proposed Pattern-Fitting Method for Wind Direction Inversion}

In this paper, a pattern-fitting method by estimating the spreading parameter $s$ or $\beta$ using two radar sites is proposed, which can be used to invert the wind direction indirectly. In fact, it is a modified form of Gurgel's method (Equation (7)), which satisfies the equation by setting the spreading parameter $s$ or $\beta$ value as undetermined and finding the appropriate spreading value for Equation (8).

$$
\left[R_{1}-\frac{G\left(\phi_{1}-\theta_{k}+\pi\right)}{G\left(\phi_{1}-\theta_{k}\right)}\right]^{2}+\left[R_{2}-\frac{G\left(\phi_{2}-\theta_{k}+\pi\right)}{G\left(\phi_{2}-\theta_{k}\right)}\right]^{2}=0,
$$

If the half-cosine 2s-power type is implemented for the inversion, Equation (8) can be written as

$$
\theta=\left\{\begin{array}{ll}
\phi_{1} \pm 2 \arctan \left(R_{1}^{\frac{1}{2 s}}\right) ; & \left(\theta_{1}^{ \pm}\right) \\
\phi_{2} \pm 2 \arctan \left(R_{2}^{\frac{1}{2 s}}\right) ; & \left(\theta_{2}^{ \pm}\right)
\end{array},\right.
$$

where the symbol " \pm " in $\theta_{i}^{ \pm}$represents " \pm " in the equations. At the sea surface, the wind direction is unique and so is the spreading parameter $s$. Even though these parameters are unknown, they can be derived from Equation (9). We take the example by setting $R_{1}<1, R_{2}<1$, as shown in Table 1 . In case of $\pi / 2<\phi_{2}-\phi_{1}<\pi$, there could be only one cross point for the two pairs of direction curves, which are the curves $\theta_{1}^{+}$and $\theta_{2}^{-}$. In case of $0<\phi_{2}-\phi_{1}<\pi / 2$, there might be only one cross point (curves $\theta_{1}^{+}$and $\theta_{2}^{-}$) or more than one cross point.

Table 1. The possible cross-points for the cosine function $\left(R_{1}<1, R_{2}<1\right)$.

\begin{tabular}{cccc}
\hline $\boldsymbol{\phi}_{2}, \boldsymbol{\phi}_{1}$ & $\boldsymbol{R}_{\mathbf{1}}, \boldsymbol{R}_{\mathbf{2}}$ & $\begin{array}{c}\text { Curves with a } \\
\text { Cross-Point }\end{array}$ & Conditions \\
\hline $0<\phi_{2}-\phi_{1}<\pi / 2$ & $R_{2}<R_{1}<1$ & $\left(\theta_{1}^{+}, \theta_{2}^{-}\right)\left(\theta_{1}^{+}, \theta_{2}^{+}\right)^{1}$ & $R_{1}>\left[\frac{\tan \left(0.5 \cdot\left(\phi_{2}-\phi_{1}\right)\right)+R_{2}^{\frac{1}{2 s}}}{1-R_{2}^{\frac{1}{2 s}} \cdot \tan \left(0.5 \cdot\left(\phi_{2}-\phi_{1}\right)\right.}\right]^{2 \mathrm{~s}}$ \\
\cline { 2 - 4 } & $R_{1}<R_{2}<1$ & $\left(\theta_{1}^{+}, \theta_{2}^{-}\right)\left(\theta_{1}^{-}, \theta_{2}^{-}\right)^{1}$ & $R_{2}>\left[\frac{\tan \left(0.5 \cdot\left(\phi_{2}-\phi_{1}\right)\right)+R_{1}^{\frac{1}{2 s}}}{1-R_{1}^{\frac{1}{2 s}} \cdot \tan \left(0.5 \cdot\left(\phi_{2}-\phi_{1}\right)\right)}\right]^{2 \mathrm{~s}}$ \\
\hline$\pi / 2<\phi_{2}-\phi_{1}<\pi$ & $R_{1}<1, R_{2}<1$ & $\left(\theta_{1}^{+}, \theta_{2}^{-}\right)$ & \\
\hline $1^{1}\left(\theta_{1}^{+}, \theta_{2}^{+}\right)$and $\left(\theta_{1}^{-}, \theta_{2}^{-}\right)$have a cross-point only when the condition is satisfied.
\end{tabular}

With the hyperbolic secant-squared spreading function, the uncertainty of the spreading parameter $\beta$ could also be determined using the pattern-fitting method and the wind direction:

$$
\theta_{i}=\phi_{i} \pm \frac{1}{2 \beta} \ln \left|\frac{1-R_{i}^{\frac{1}{2}} e^{-\beta \cdot \pi}}{R_{i}^{\frac{1}{2}} e^{\beta \cdot \pi}-1}\right|(i=1 \text { or } 2) .
$$


The ratio $R_{i}$ can be easily calculated from the radar's Doppler spectrum. As discussed before, the spreading parameter $\beta$ must meet Equation (11).

$$
\frac{\operatorname{sech}^{2}\left(\beta_{i, \min } \cdot \pi\right)}{\operatorname{sech}^{2}\left(\beta_{i, \min } \cdot 0\right)}=R_{i}
$$

Hence,

$$
\beta_{i, \text { min }}^{ \pm}=\frac{1}{\pi} \ln \left[\left(\frac{1}{R_{i}}\right)^{1 / 2} \pm \frac{1}{\left(R_{i}-1\right)^{1 / 2}}\right]
$$

if $R_{i}>1$, we also need to make sure the direction can cover the entire range of $[-\pi, \pi]$; thus, when $|\theta-\phi|=\pi$ or $-\pi)$,

$$
\frac{\operatorname{sech}^{2}\left(\beta_{i, \min } \cdot 0\right)}{\operatorname{sech}^{2}\left(\beta_{i, \min } \cdot \pi\right)}=R_{i} .
$$

Hence,

$$
\beta_{i, \min }^{ \pm}=\frac{1}{\pi} \ln \left[R_{i}^{1 / 2} \pm\left(R_{i}-1\right)^{1 / 2}\right]
$$

Figure 2 shows the results of $\theta_{i}^{ \pm}(i=1,2)$ without the limitation of $\beta_{\min }$, that is to say, with the given value of the ratio $R_{i}$ that not only the direction in the range $[-\pi, \pi]$ can satisfy, but also in which the angle exceeds the range of $[-\pi, \pi]$. Due to the aperiodicity of $\operatorname{sech}^{2}(\beta \cdot \theta)$, the limitation of the spreading parameter $\beta$ is computed according to Equation (14).

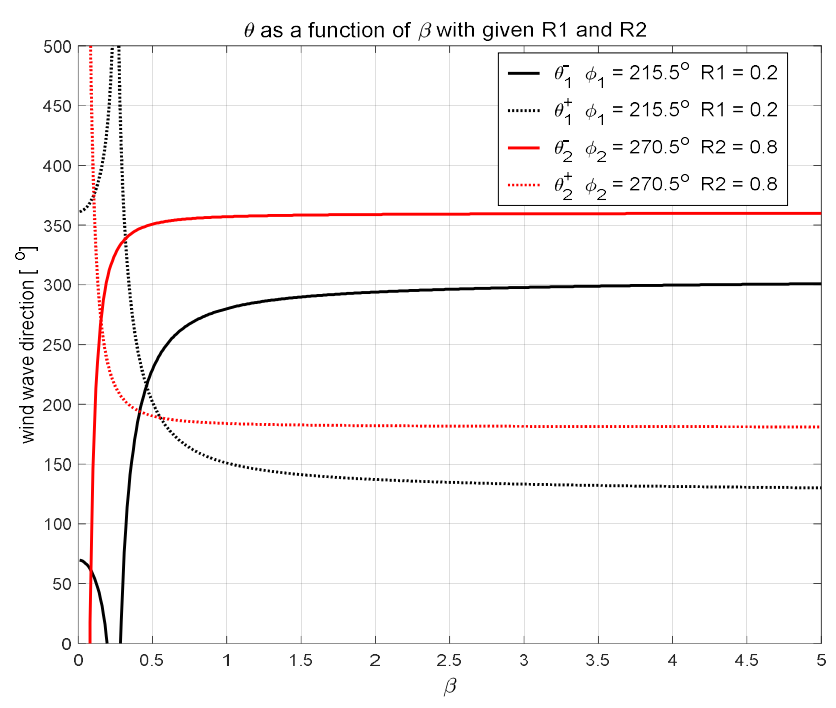

Figure 2. $\theta$ as a function of $\beta$ with given $R_{1}$ and $R_{2}$.

The cross-point of two pairs of curves $\left(\theta_{1}^{ \pm}\right.$and $\theta_{2}^{ \pm}$) gives the value of $\beta$ and the wind direction $\theta$. As indicated in Figure $2, \beta_{0}=0.5732$ and the wind direction $\theta_{0}=188.3^{\circ}$. Figure 3 shows an example of the spreading parameter $\beta_{0}$ and the direction $\theta_{0}$ derived from the ratios $R_{1}=0.2$ and $R_{2}=0.8$ and $\phi_{1}=215.5^{\circ}$ and $\phi_{2}=270.5^{\circ}$.

When $R_{i} \geq 1$ is considered, the angle between the radio beam direction and the wind direction at $\beta_{i, \min }$ is given as follows:

$$
\begin{aligned}
\theta_{i, \beta_{\min }} & =\frac{1}{2 \beta_{i, \min }} \ln \left|\frac{1-R_{i}^{1 / 2} \exp \left(-\beta_{i, \min } \cdot \pi\right)}{R_{i}^{1 / 2} \exp \left(\beta_{i, \min } \cdot \pi\right)-1}\right| \\
& =\left\{\begin{array}{cl}
-\pi & \left(R_{i}>1\right) \\
0 & \left(R_{i}<1\right) \\
-\pi / 2 & \left(R_{i}=1\right)
\end{array}\right.
\end{aligned}
$$


Table 2 gives the relationship of $\beta_{1, \min }$ and $\beta_{2, \min }$ and the wind direction $\theta$ at the start point $\left(\beta_{i, \min }\right)$.

Table 2. Ratio and the spreading parameter $\beta_{\min }\left(R_{1}<1, R_{2}<1\right)$.

\begin{tabular}{|c|c|c|}
\hline$R_{1}$ and $R_{2}$ & $\beta_{i, \min }$ & $\theta_{i, \beta}\left(\beta=\beta_{\min }\right)$ \\
\hline$R_{1} \geq R_{2}>1$ & $\beta_{1, \min } \geq \beta_{2, \min }$ & $\theta_{1}^{ \pm}=\phi_{1} \pm \pi ; \theta_{2}^{ \pm}=\phi_{2} \pm \pi$ \\
\hline$R_{2}>R_{1}>1$ & $\beta_{1, \min }<\beta_{2, \text { min }}$ & $\theta_{1}^{ \pm}=\phi_{1} \pm \pi ; \theta_{2}^{ \pm}=\phi_{2} \pm \pi$ \\
\hline$R_{1}^{-1} \geq R_{2}>1$ & $\beta_{1, \min } \geq \beta_{2, \min }$ & $\theta_{1}^{ \pm}=\phi_{1} ; \theta_{2}^{ \pm}=\phi_{2} \pm \pi$ \\
\hline$R_{2}>R_{1}^{-\overline{1}}>1$ & $\beta_{1, \min }<\beta_{2, \min }$ & $\theta_{1}^{ \pm}=\phi_{1} ; \theta_{2}^{ \pm}=\phi_{2} \pm \pi$ \\
\hline$R_{1} \geq R_{2}^{-1}>1$ & $\beta_{1, \min } \geq \beta_{2, \min }$ & $\theta_{1}^{ \pm}=\phi_{1} \pm \pi ; \theta_{2}^{ \pm}=\phi_{2}$ \\
\hline$R_{2}^{-1}>R_{1}>1$ & $\beta_{1, \min }<\beta_{2, \min }$ & $\theta_{1}^{ \pm}=\phi_{1} \pm \pi ; \theta_{2}^{ \pm}=\phi_{2}$ \\
\hline$R_{1}<R_{2}<1$ & $\beta_{1, \min } \geq \beta_{2, \min }$ & $\theta_{1}^{ \pm}=\phi_{1} ; \theta_{2}^{ \pm}=\phi_{2}$ \\
\hline$R_{2}<R_{1}<1$ & $\beta_{1, \min }<\beta_{2, \min }$ & $\theta_{1}^{ \pm}=\phi_{1} ; \theta_{2}^{\ddagger}=\phi_{2}$ \\
\hline$R_{1}=1$ or $R_{2}=1$ & & $\theta_{1}^{ \pm}=\phi_{1} \pm \frac{\pi}{2}[2]$ \\
\hline
\end{tabular}

[1] There is no minimum value of $\beta_{i}$ (where $i$ makes $R_{i}=1$ ). ${ }^{[2]}$ Where $i$ makes $R_{i}=1$, the other $\theta_{i}$ can refer to the conditions above.

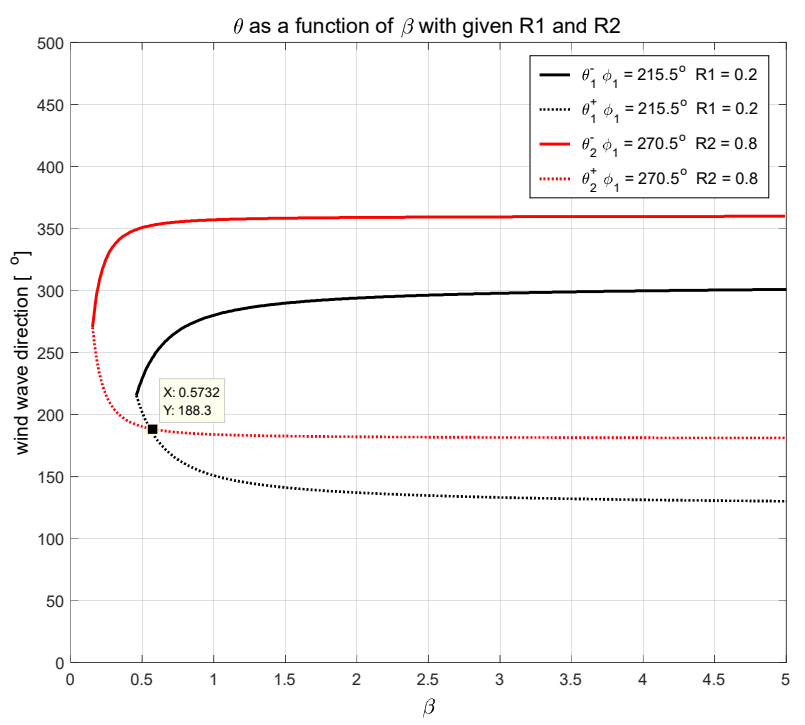

Figure 3. $\theta$ as a function of $\beta$ with given $R_{1}$ and $R_{2}\left(\beta>\beta_{\min }\right)$.

Compared with the half-cosine function, the hyperbolic secant function can give a unique solution for $\left(\beta_{0}, \theta_{0}\right)$. For example, one direction curve $\theta_{2}^{-}$will not have one cross-point with the curve $\theta_{1}^{+}$and some other possible cross-points with the curve $\theta_{1}^{-}$because the start point of the direction curves $\beta_{i \text {,min }}$ is not fixed at the value of zero, which also changes with the ratio $R_{i}$ and makes a unique solution possible. The hyperbolic secant function gives a unique solution for wind direction determination and the spreading parameter $\beta$. That is to say, there can be only one cross-point of the two pairs of wind directions $\theta_{1}^{ \pm}$and $\theta_{2}^{ \pm}$. The proof is given below.

We take $R_{\mathrm{i}}<1$ as an example and the radio beam direction is defined as $0<\phi_{2}-\phi_{1}<\pi$ on the premise of $\pi / 2<\phi_{2}-\phi_{1}<\pi$ and the curve $\theta_{1, \beta \rightarrow \infty}^{+}=\theta_{1}+\pi<\phi_{2}$. Likewise, there is no cross-point for $\theta_{1}^{+}$and $\theta_{2}^{+}$either. If $0<\phi_{2}-\phi_{1}<\pi / 2$, there might be a cross-point for the curves $\theta_{1}^{+}$and $\theta_{2}^{+}$and the wind direction $\theta$.

$$
\begin{gathered}
\theta=\phi_{1}+\frac{1}{2 \beta} \ln \left|\frac{1-R_{1}^{1 / 2} e^{-\beta \cdot \pi}}{R_{1}^{1 / 2} e^{\beta \cdot \pi}-1}\right| \\
=\phi_{2}+\frac{1}{2 \beta} \ln \left|\frac{1-R_{2}^{1 / 2} e^{-\beta \cdot \pi}}{R_{2}^{1 / 2} e^{\beta \cdot \pi}-1}\right|
\end{gathered}
$$

where $\beta \geq \max \left(\beta_{1, \min }, \beta_{2, \min }\right)$ as $\phi_{2}-\phi_{1}>0$; thus, $\mathrm{e}^{2 \beta\left(\phi_{2}-\phi_{1}\right)}>1$ is defined. 
In the case of $R_{1} \geq R_{2}$,

$$
\frac{\left(1-e^{\beta \pi} R_{2}^{1 / 2}\right)\left(1-e^{-\beta \pi} R_{1}^{1 / 2}\right)}{\left(1-e^{\beta \pi} R_{1}^{1 / 2}\right)\left(1-e^{-\beta \pi} R_{2}^{1 / 2}\right)} \leq 1 \quad\left(\beta \geq \beta_{2, \min }\right) .
$$

Therefore, there is no cross-point when $R_{1} \geq R_{2}$, and there might be a cross-point when $R_{1}<R_{2}$. According to Table 2 , we know that $\beta_{1, \min } \geq \beta_{2, \min }$; hence, $\max \left(\beta_{1, \min }, \beta_{2, \min }\right)=\beta_{1, \text { min }}$.

Equation (17) is difficult to solve; therefore, for the curves $\theta_{i}^{ \pm}$, the method of monotonicity is implemented to prove that there can only be one cross-point for the curves that provides a unique solution for the spreading parameter and wind direction. We define the following:

$$
\begin{gathered}
F_{1}(\beta)=e^{2 \beta\left(\phi_{2}-\phi_{1}\right)} ; \\
F_{2}(\beta)=\frac{\left(1-e^{\beta \pi} R_{2}^{1 / 2}\right)\left(1-e^{-\beta \pi} R_{1}^{1 / 2}\right)}{\left(1-e^{\beta \pi} R_{1}^{1 / 2}\right)\left(1-e^{-\beta \pi} R_{2}^{1 / 2}\right)} .
\end{gathered}
$$

Thus, we have

$$
\begin{gathered}
\frac{\partial F_{1}}{\partial \beta}=e^{2 \beta\left(\phi_{2}-\phi_{1}\right)} \cdot 2\left(\phi_{2}-\phi_{1}\right)>0 ; \\
\frac{\partial F_{2}}{\partial \beta}=\frac{\pi\left(R_{1}^{1 / 2}-R_{2}^{1 / 2}\right)}{\left[1-e^{\beta \pi} R_{1}^{1 / 2}\right]^{2}\left[1-e^{-\beta \pi} R_{2}^{1 / 2}\right]^{2}} \cdot \frac{\left[1+\left(R_{1} R_{2}\right)^{1 / 2}\right]\left(e^{-\beta \pi}+e^{\beta \pi}\right)-2\left(R_{1}^{1 / 2}+R_{2}^{1 / 2}\right)}{\left(1-e^{\beta \pi} R_{1}^{1 / 2}\right)^{2}\left(1-e^{-\beta \pi} R_{2}^{1 / 2}\right)^{2}} ; \\
\because\left(1-e^{\beta \pi} R_{1}^{1 / 2}\right)^{2}\left(1-e^{-\beta \pi} R_{2}^{1 / 2}\right)^{2}>0 \text { and } R_{1}<R_{2} ; \\
\therefore \frac{\pi\left(R_{1}^{1 / 2}-R_{2}^{1 / 2}\right)}{\left(1-e^{\beta \pi} R_{1}^{1 / 2}\right)^{2}\left(1-e^{-\beta \pi} R_{2}^{1 / 2}\right)^{2}}<0 ;
\end{gathered}
$$

and

$$
e^{-\beta \pi}+e^{\beta \pi}>2 \quad(\beta \neq 0) .
$$

As such, we have

$$
\begin{aligned}
& {\left[1+\left(R_{1} R_{2}\right)^{1 / 2}\right]\left(e^{-\beta \pi}+e^{\beta \pi}\right)-2\left(R_{1}^{1 / 2}+R_{2}^{1 / 2}\right)} \\
& >2\left[\left(1+\left(R_{1} R_{2}\right)^{1 / 2}\right)-R_{1}^{1 / 2}-R_{2}^{1 / 2}\right] \\
& =2\left[\left(1-R_{1}^{1 / 2}\right)\left(1-R_{2}^{1 / 2}\right)\right]>0
\end{aligned}
$$

Therefore,

$$
\frac{\partial F_{2}}{\partial \beta}<0 .
$$

For example, if we set $\phi_{2}=250.5^{\circ}, \phi_{1}=205.5^{\circ}$, and $R_{1}=0.3$, we calculate the threshold of $R_{2, \min }=0.5272$. Thus, if $R_{2}>R_{2, \min }$, the curves $\theta_{2}^{+}$and $\theta_{1}^{+}$will have a cross-point, and when $R_{2}=R_{2, \min }$, the cross-point will be at $\left(\beta_{1, \min }, \phi_{1}\right)$. In Figure $4 \mathrm{a}$, the curves $F_{1}$ and $F_{2}$ are given to illustrate the strictly monotone increase of $F_{1}(\beta)=e^{2 \beta\left(\phi_{2}-\phi_{1}\right)}$ and the strictly monotone decrease of $\mathrm{F}_{2}(\beta)$ as given in Equation (19). As discussed before, the $\beta \geq \max \left(\beta_{1, \min }, \beta_{2, \min }\right)$; here, $\beta_{\min }=\beta_{1, \min }$. Figure $4 \mathrm{~d}$ illustrates the cross-point of curves $\theta_{1}^{+}$and $\theta_{2}^{+}$; the cross-point locates at the start point of the curve of $\theta_{1}^{+}$.

If $R_{2}>R_{2, \min }$, we define $R_{2}=0.7272$. Figure $4 \mathrm{~b}$ gives the curve of $F_{1}(\beta)$ and $F_{2}(\beta)$. Because of the characteristic of the monotone variance of $F_{1}(\beta)$ and $F_{2}(\beta)$, and as we proved at $\beta_{\min }, \mathrm{F}_{1}\left(\beta_{\min }\right)<$ $F_{2}\left(\beta_{\min }\right)$ and when $\beta \rightarrow+\infty, F_{1}(\beta \rightarrow \infty)>F_{2}(\beta \rightarrow \infty)$, these two curves just have one cross-point. As shown in Figure 4e, the curves $\theta_{1}^{+}$and $\theta_{2}^{+}$will have only one cross-point. Now that the cross-point is figured out, we have $\beta=0.478$ and the wind wave direction $\theta=175^{\circ}$.

If $R_{2}<R_{2, \min }$, we define $R_{2}=0.3272$. As shown in Figure $4 \mathrm{f}$, the curves $\theta_{1}^{+}$and $\theta_{2}^{+}$will not have a cross-point. Figure $4 \mathrm{c}$ gives the curve of $F_{1}(\beta)$ and $F_{2}(\beta)$. Because of the characteristic of the monotone variance of $F_{1}(\beta)$ and $F_{2}(\beta)$ and as we proved at $\beta_{\min }, F_{1}\left(\beta_{\min }\right)>F_{2}\left(\beta_{\min }\right)$ and when 
$\beta \rightarrow+\infty, F_{1}(\beta \rightarrow \infty)>F_{2}(\beta \rightarrow \infty)$, these two curves have no cross-point. Figure $4 \mathrm{f}$ shows the curves $\theta_{1}^{+}$and $\theta_{2}^{+}$. The cross-point is located at the curve $\theta_{1}^{-}$and $\theta_{2}^{+}$, but not at the curve $\theta_{1}^{+}$and $\theta_{2}^{+}$. Now that the cross-point is also figured out, we have $\beta=0.44$ and the wind wave direction $\theta=226^{\circ}$.

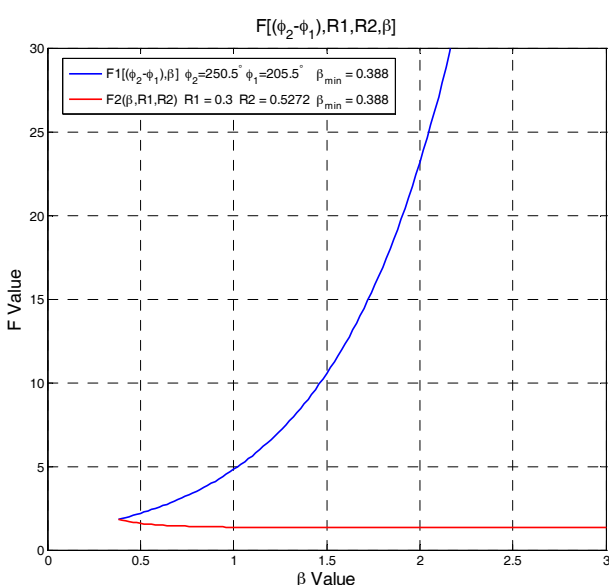

(a)

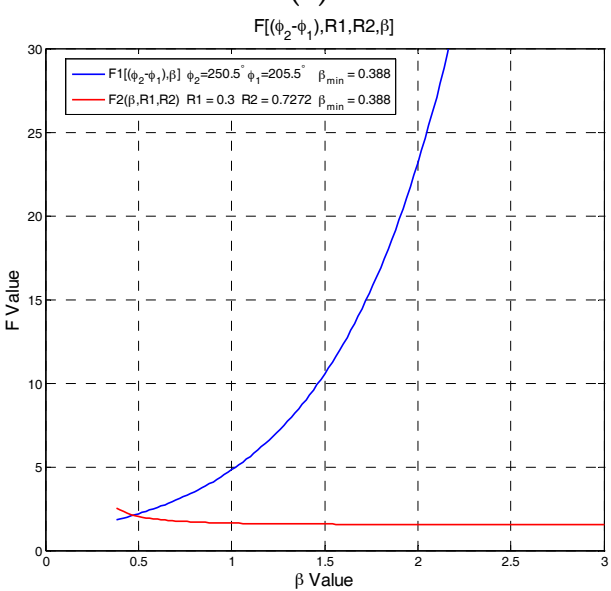

(c)

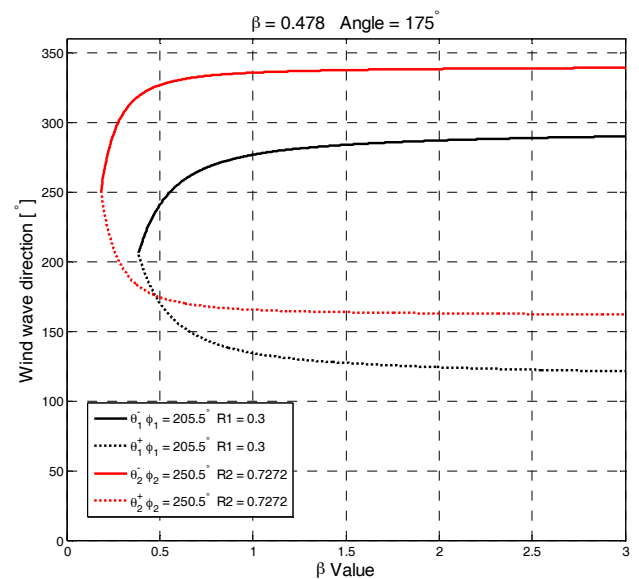

(e)

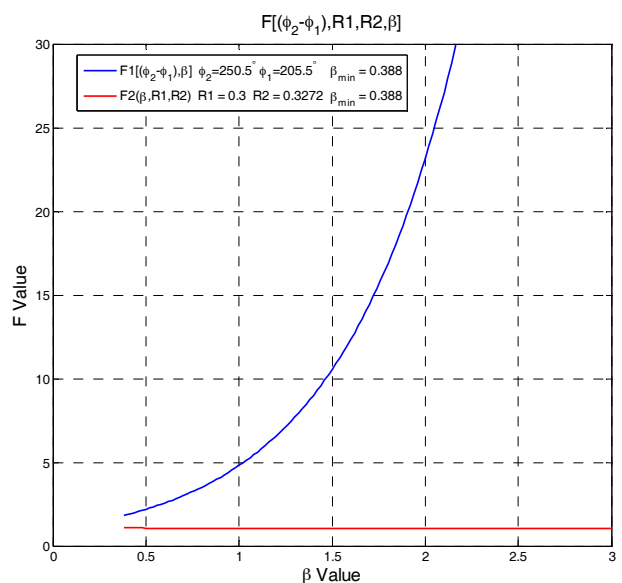

(b)

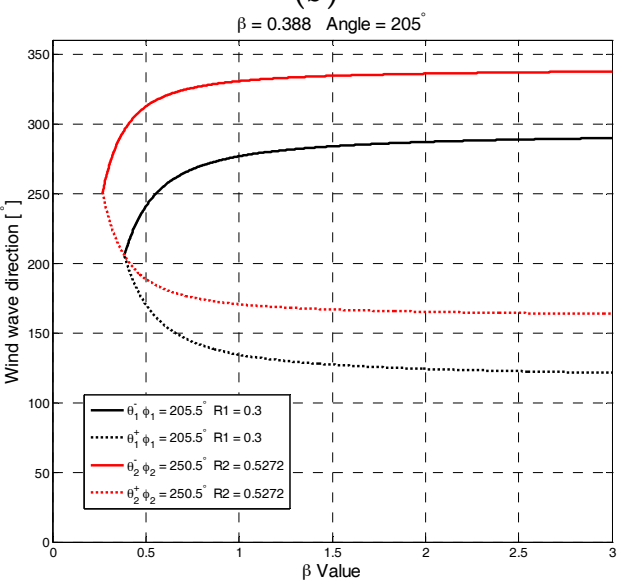

(d)

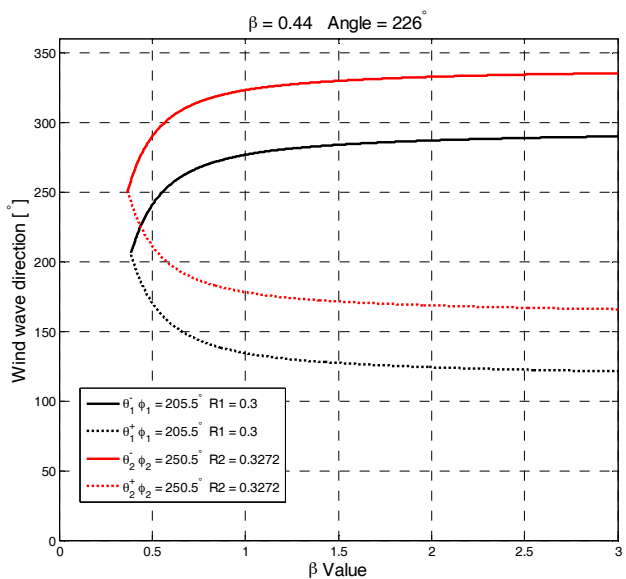

(f)

Figure 4. The threshold for $R_{2}$ having a cross-point for the curves $\theta_{1}^{+}$and $\theta_{2}^{+}$. (a) Curves of $F_{1}$ and $F_{2}$ $\left(R_{2}=R_{2, \min }\right)$; (b) curves of $F_{1}$ and $F_{2}\left(R_{2}>R_{2, \min }\right)$; (c) curves of $F_{1}$ and $F_{2}\left(R_{2}<R_{2, \min }\right)$; (d) curves of $\theta_{1}^{ \pm}$ and $\theta_{2}^{ \pm}\left(R_{2}=R_{2, \min }\right)$; (e) curves of $\theta_{1}^{ \pm}$and $\theta_{2}^{ \pm}\left(R_{2}>R_{2, \min }\right)$; and (f) curves of $\theta_{1}^{ \pm}$and $\theta_{2}^{ \pm}\left(R_{2}<R_{2, \min }\right)$. 


\subsection{Wind Direction and Wavelength of Resonant Waves}

When wind blows over the sea surface, the energy is transferred from the air to the water via wind-wave interaction, and, if the wind continuously impacts on the sea surface, the wave grows from short waves to long waves. The long waves have some time delay in responding to wind changes. Due to an air pressure difference, the wind might change its direction (it takes hours for a wind direction change); thus, the short waves are more sensitive to a wind direction change, while longer waves take more time for wave growth. Figure 5 gives WAM (wave model) data, including wind direction and wave direction at each frequency bin, as well as the mean wave direction. In this example, the direction of short waves $(0.54 \mathrm{~Hz})$ is nearly identical to the wind direction, while the longer waves are offset by nearly $20^{\circ}$. Another in situ measurement is illustrated during the experiment in Italy, in which a meteorological buoy measured the wind direction, and a wave-rider buoy measured the wave direction at each frequency bin. All these results indicate that the measurements of short waves are more reliable than those of long waves.

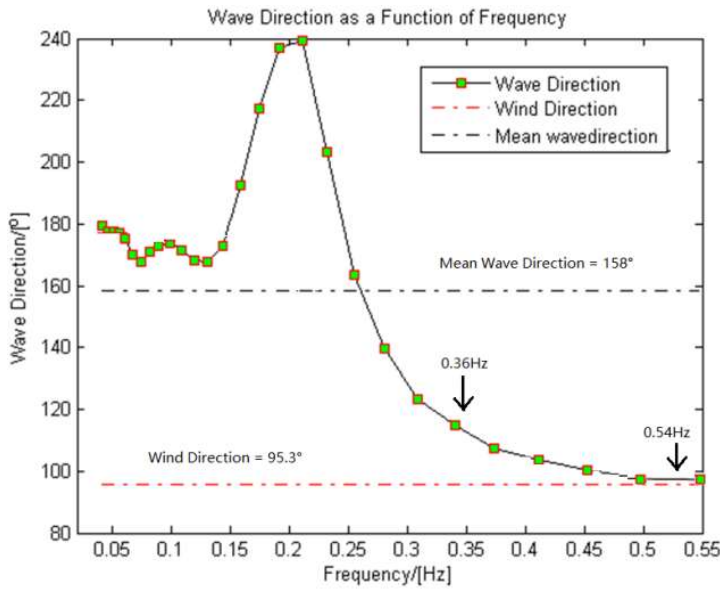

(a)

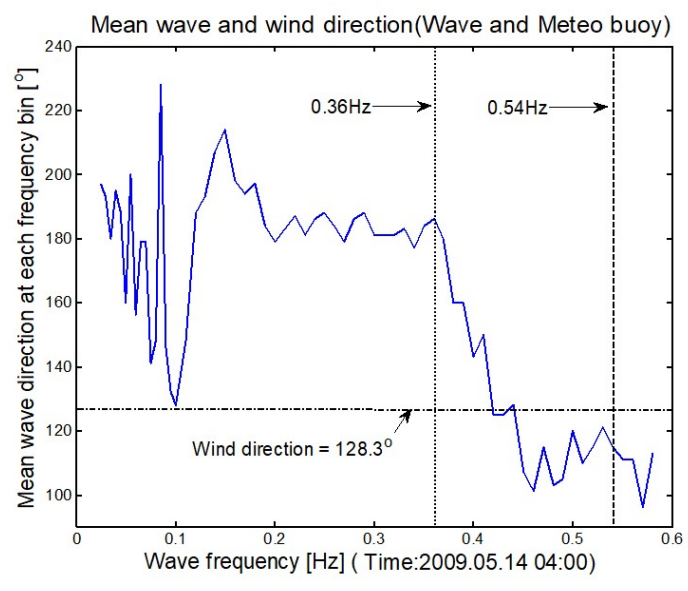

(b)

Figure 5. Wind direction and wave direction at each frequency bin. (a) WAM (wave model) data; (b) in situ measurement at the Thyrrenian Sea, Italy.

\subsection{Wind Direction Inversion and Wind Speed}

For wind direction inversion from HF radar backscatter measurements, if the wind speed is low, it means that the sea surface is relatively calm. In this case, the wind direction is meaningless, and although the wind direction can be inverted from radar backscatter signals, the result is quite random. Thus, in this case, we set as a threshold of wind speed for wind direction inversion as a wind speed higher than $3 \mathrm{~m} / \mathrm{s}$, so that the wind direction would be relatively reliable. Under high-wind conditions, the resonant waves (i.e., wave frequencies at $0.54 \mathrm{~Hz}$ and $0.36 \mathrm{~Hz}$ ) are both fully developed, and, in this case, the wind direction measurements are also reliable. However, under low-wind conditions, the short resonant waves are fully developed while the longer resonant waves are not. If the wind starts changing its direction, the measurement of the wind direction from longer resonant waves will not be as reliable as that from short resonant waves.

\section{Experiments and Results}

\subsection{Introduction to Radar Experiments and Wind Conditions}

Two radar experiments were carried out to verify the proposed wind direction inversion method. The radar frequency and wind conditions were quite different in each experiment. With different radar frequencies and wind conditions, we could study the influencing factor for wind direction inversion. 
One experiment was the Fedje experiment within the EuroROSE (European radar ocean sensing) project, which was carried out in Norway (Fedje and Lyngoy) from February to April in 2000. The radar operating frequency was $27.68 \mathrm{MHz}$ and the coverage was about $40 \times 40 \mathrm{~km}^{2}$. The beam formation for two radar sites was achieved using a 16-element linear receiving antenna array. Each radar covers a sector of $120^{\circ}$. One wave buoy was deployed nearly $6 \mathrm{~km}$ off the coast, which provided the wind measurements and the directional wave spectrum measurements. The map for the experiment is illustrated in Figure 6a. The Ligurian Sea experiment was conducted to investigate HF radar wind inversion in Italy from April to September 2009. The radars were placed at Palmaria and Rossore with a distance of $50 \mathrm{~km}$. A meteorological buoy and a wave-rider buoy were deployed between radar sites about $30 \mathrm{~km}$ offshore (43.876N, 9.873E). The meteorological buoy provides in situ wind direction and speed measurements at the sea surface and the wave-rider buoy provides in situ wave power density and wave direction measurements, as shown in Figure $6 \mathrm{~b}$.

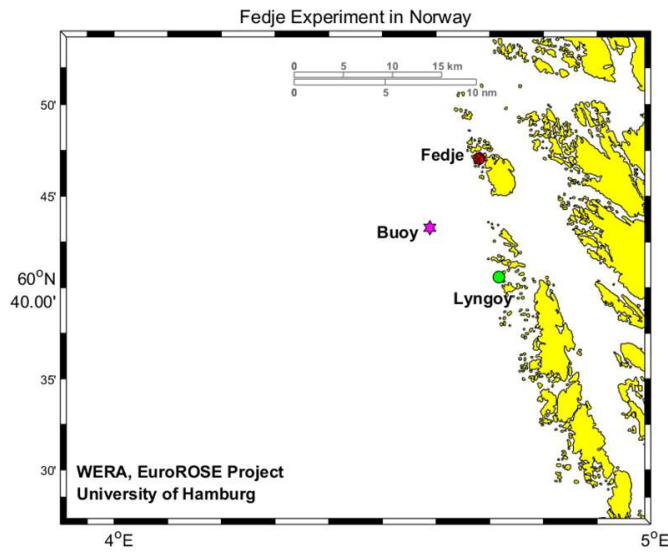

(a)

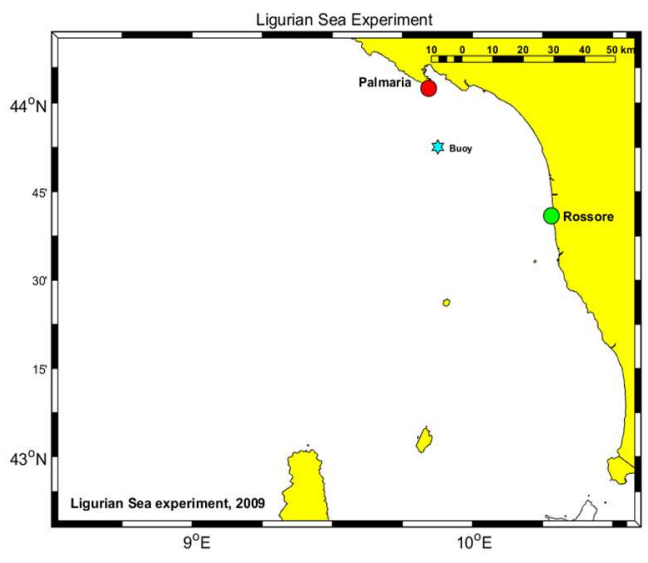

(b)

Figure 6. The deployments of radar sites and in situ buoys: (a) the Fedje experiment in Norway; (b) the Ligurian Sea experiment in Italy.

Table 3 gives a list of radar frequencies and the corresponding Bragg wavelengths and frequencies. The property of wave growth rate and directional spreading is quite different due to their wavelengths, which also produces the variance in wind direction inversion from HF radar backscatter signals.

Table 3. Radar frequencies and corresponding Bragg waves.

\begin{tabular}{ccc}
\hline Radar Frequency (MHz) & Bragg Wave Frequency (Hz) & Bragg Wave Length $\mathbf{( m )}$ \\
\hline 12 & 0.3534 & 12.5 \\
27.68 & 0.5368 & 5.419 \\
\hline
\end{tabular}

The statistics of wind speed are shown in Figure 7. The wind speed at the Norwegian Sea is higher than that at the Ligurian Sea; $67.4 \%$ of the wind records were higher than $5 \mathrm{~m} / \mathrm{s}$. During the Ligurian Sea experiment, only $18.9 \%$ of the wind records exceeded $5 \mathrm{~m} / \mathrm{s}$. In other words, the Fedje experiment was done under higher-wind conditions compared to the Ligurian Sea experiment. 


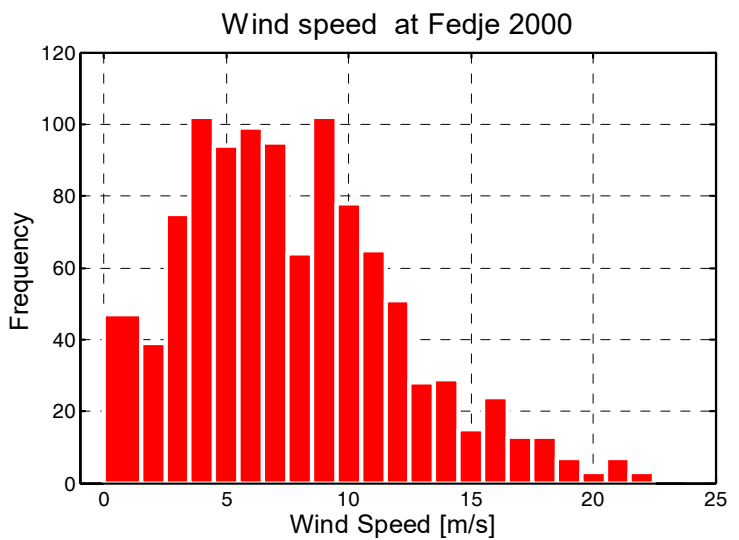

(a)

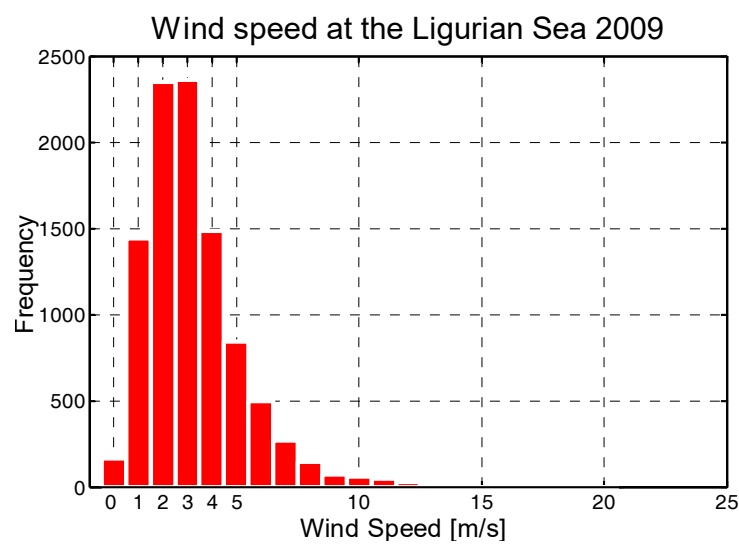

(b)

Figure 7. Statistics of wind speed measurements by the anemometer: (a) Norway experiment; (b) Ligurian Sea experiment.

The wave-rider buoy provides the mean wave direction measurement at each frequency bin. Regarding the two radar frequencies, the comparison of the mean wave direction at the corresponding Bragg frequencies and the anemometer-measured wind direction are presented in Figure 8a,b. As seen in these figures, the wind direction and the mean direction of Bragg resonant waves are not always identical, especially when the radar operates at a lower frequency (longer Bragg resonant waves). In addition to that, the wind speed is also an important factor. During the Fedje experiment, the wind speed was higher and the mean wave direction agreed with the wind direction well, while, during the Ligurian Sea experiment, the wind speed was low and the turbulence of the air flow at the sea surface brought more difficulties for wind direction measurement.

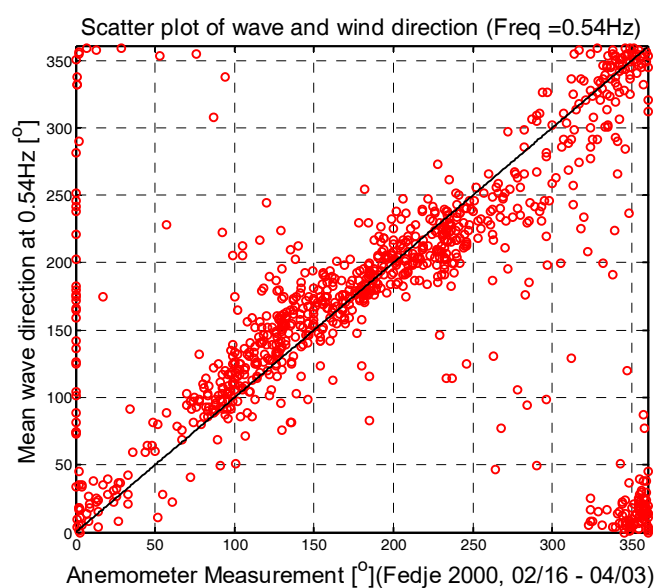

(a)

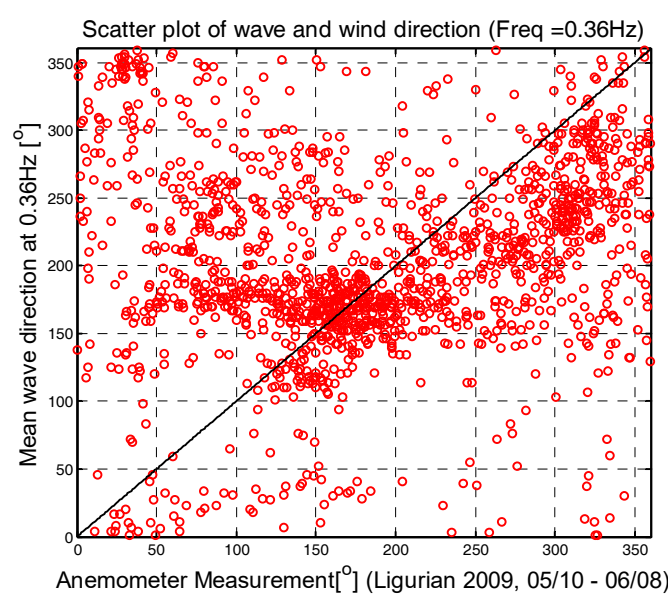

(b)

Figure 8. Mean wave direction at Bragg frequencies (wave-rider) vs. wind direction (anemometer) during the two experiments, (a) Norway experiment, (b) Ligurian Sea experiment.

\subsection{Result of Experiments}

In M. Donelan's [6] work, he suggested that a hyperbolic function gives a better approximation of wave directional distribution in nature. The minimum value of $\beta\left(\beta_{\min }\right)$ depends on the power ratio of Bragg peaks, which makes $\beta_{\min }>0$, while the minimum spreading parameter $s$ in the half-cosine 2 s-power function is zero $\left(s_{\min }=0\right)$. In some extreme cases, there might be more than one cross-point for the directional curves. Therefore, in this paper, only the hyperbolic secant-squared function was used for wind direction inversion and it gives a unique mathematical solution. 
The scatter plot of the wind direction measurement between the radar measurement and the anemometer measurement is presented in Figure 9a. Considering low-wind-speed conditions, especially when the wind speed is near to zero, the wind direction is easily disturbed by the turbulence of air flow. In this case, the wind direction measurement is meaningless. Hence, in this figure, wind direction comparisons are given when the wind speed is higher than $3 \mathrm{~m} / \mathrm{s}$. The result using the LSM method is shown in Figure 9b, in which the half-cosine 2s-power function is used and the spreading parameter $s=1$. From the comparison of the two methods, the new method presents a better result than the traditional one.

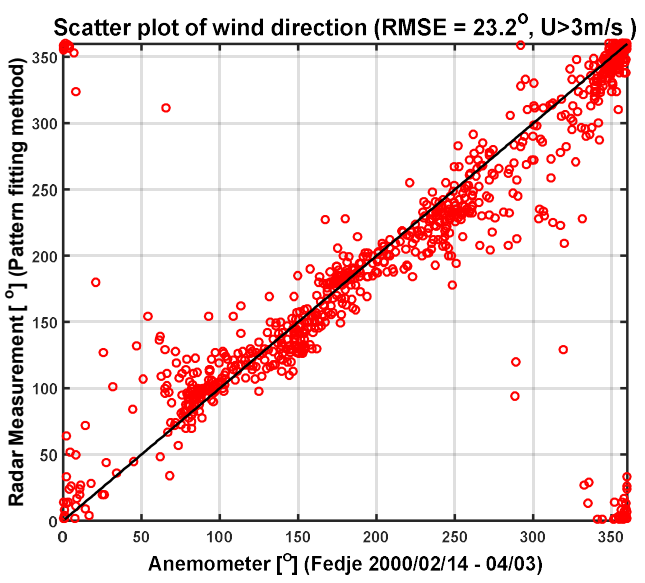

(a)

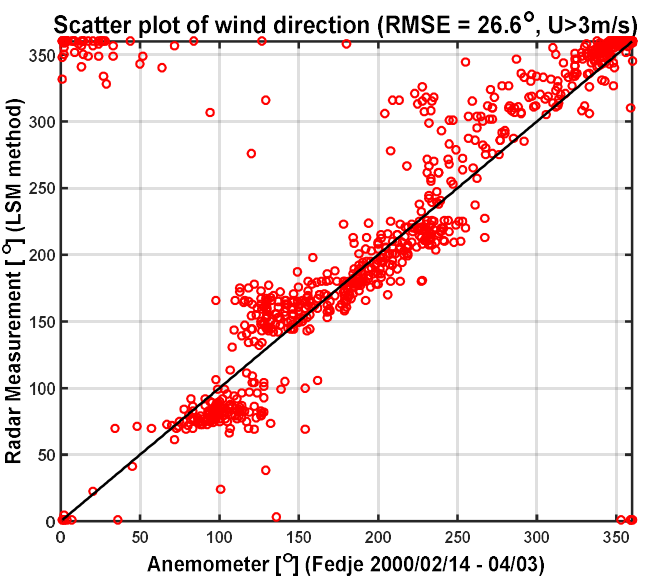

(b)

Figure 9. Comparison of wind direction measured by radar using the pattern-fitting method and the least-square minimum (LSM) method during the Fedje experiment $\left(U_{10}>3 \mathrm{~m} / \mathrm{s}\right)$. (a) Pattern-fitting method; (b) LSM method provided by Gurgel.

The error in wind direction measurement also depends on the wind speed. The radar-derived wind direction is the direction of the Bragg resonant waves. At high wind speeds, the direction of the Bragg waves agrees well with the wind direction; therefore, the wind direction measurement is more accurate if the wind speed is high. Table 4 presents the root-mean-square (RMS) error of the wind direction inversion related to the wind speed conditions during the Fedje experiment.

Table 4. Comparison of the root-mean-square (RMS) error of the wind direction related to wind speed using the pattern-fitting and least-square minimum (LSM) methods in the Fedje experiment.

\begin{tabular}{ccccc}
\hline \multicolumn{5}{c}{ RMS Error for Wind Direction Measurements $\mathbf{(}^{\circ}$ ) } \\
\hline \multirow{2}{*}{ Comparison of inversion method } & Wind Speed $(\mathrm{m} / \mathrm{s})$ & \multicolumn{2}{c}{ Wind Speed Range $(\mathrm{m} / \mathrm{s})$} \\
& $\mathrm{U}>3$ & $0<\mathrm{U} \leq 3$ & $3<\mathrm{U} \leq 10$ & $\mathrm{U}>10$ \\
\hline Pattern-fitting method & 23.2 & 72.5 & 25.8 & 12.5 \\
\hline LSM method & 26.6 & 75.9 & 29.5 & 14.8 \\
\hline
\end{tabular}

During the Ligurian Sea experiment, the radar frequency is $12 \mathrm{MHz}$, the wavelength of Bragg waves is $12 \mathrm{~m}$, and the Bragg wave frequency is $0.3534 \mathrm{~Hz}$. The wavelength of the Bragg waves is nearly twice that measured during the Fedje experiment. For longer Bragg waves, the directional spreading pattern might not be as regular as that of the short waves, and the wave response (time and amplitude) to the changes in wind is different as well. The scatter plot of wind direction measurements using the pattern-fitting method is shown in Figure 10a and the result of the LSM method is given in Figure 10b. 


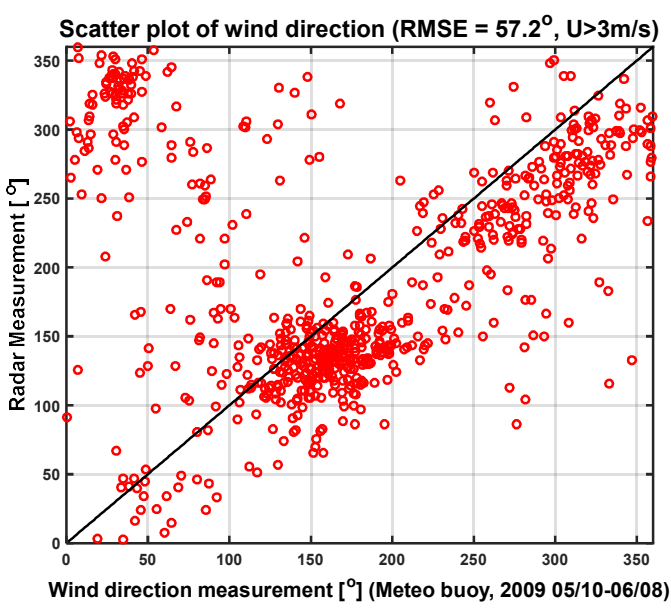

(a)

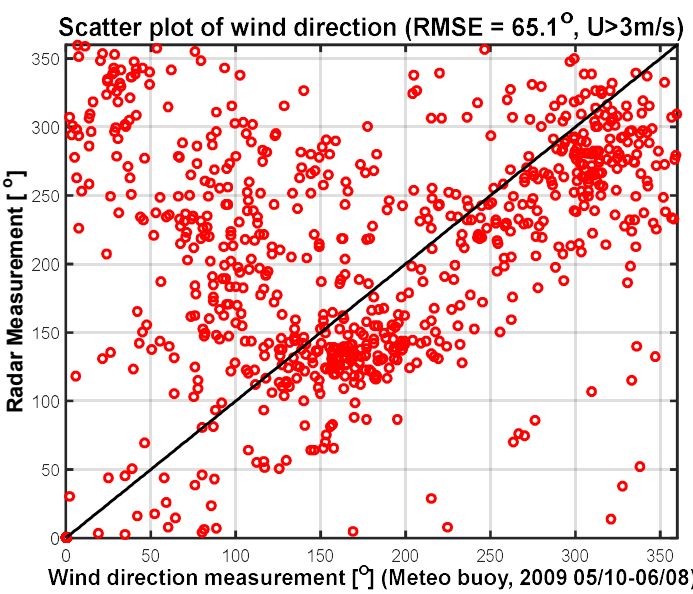

(b)

Figure 10. Comparison of wind direction measured by radar using the pattern-fitting method and the LSM method during the Ligurian Sea experiment $\left(U_{10}>3 \mathrm{~m} / \mathrm{s}\right)$. (a) Pattern-fitting method; (b) LSM method.

The RMS error analysis is presented in Table 5. As seen in the table, for longer Bragg resonant waves, the pattern-fitting method gives a better result than the conventional LSM method.

Table 5. Comparison of the RMS error of the wind direction related to wind speed using the pattern-fitting and LSM methods in the Ligurian Sea experiment.

\begin{tabular}{ccccc}
\hline \multicolumn{5}{c}{ RMS Error for Wind Direction Measurements $\mathbf{(}^{\circ}$ ) } \\
\hline \multirow{2}{*}{ Comparison of inversion method } & Wind speed $(\mathrm{m} / \mathrm{s})$ & \multicolumn{3}{c}{ Wind speed range $(\mathrm{m} / \mathrm{s})$} \\
& $\mathrm{U}>3$ & $0<\mathrm{U} \leq 3$ & $3<\mathrm{U} \leq 10$ & $\mathrm{U}>10$ \\
\hline Pattern-fitting method & 57.2 & 80.3 & 57.6 & 20.4 \\
\hline LSM method & 65.1 & 84.6 & 64.7 & 24.1 \\
\hline
\end{tabular}

\section{Conclusions}

This paper introduces a pattern-fitting method for wind direction inversion from HF radar backscatter signals by estimating the wind-wave directional spreading parameter, which provides a unique solution for the spreading parameter and wind direction. In order to verify the wind direction inversion under different wind conditions and at different radar frequencies, two radar experiments were introduced. The Fedje experiment was carried out in Norway, where the operating frequency was $27.68 \mathrm{MHz}$ with corresponding Bragg waves at a frequency of $0.54 \mathrm{~Hz}$, and the wind speed was relatively high (higher than $5 \mathrm{~m} / \mathrm{s} 67.4 \%$ of the time). The Ligurian Sea experiment was carried out in Italy, where the operating frequency was $12 \mathrm{MHz}$ with corresponding Bragg waves at a frequency of $0.36 \mathrm{~Hz}$, and the wind speed was relatively low (higher than $5 \mathrm{~m} / \mathrm{s} 18.9 \%$ of the time). With the two experiments, we can draw several conclusions.

The proposed pattern-fitting method provides measurements that are in better agreement with in situ measurements at different radar operating frequencies and also in different wind conditions, which makes the new method more reliable.

We also conclude that, for wind direction measurements from HF radar backscatter, the accuracy strongly depends on the radar frequency. When the radar works at a higher frequency, the wavelength of the Bragg resonant waves is shorter; short Bragg resonant waves are more sensitive to a change in wind field. Thus, for wind direction measurements from HF radar backscatter signals, a radar with a higher operating frequency leads to measurements that are in good agreement with in situ measurements. 
From the measurement of wind speed using buoys, we can also conclude that, under higher-wind conditions, the inversion of wind direction is better. However, we could not choose the weather during the experiment; we just found the rules for wind direction inversion under different wind conditions.

Therefore, for wind field inversion in coastal areas, the choice of radar frequency is an important issue. A radar at higher frequencies covers a smaller area, but brings a higher accuracy of wind field inversion. Users should balance radar measuring accuracy with radar coverage.

Author Contributions: W.S. conceived and performed the calculations and data analysis. K.-W.G. provided beneficial discussion and provided the radar and in situ data, as well as some WAM model data. W.S. wrote and revised the manuscript.

Funding: This research was funded by the National Natural Science Foundation in China (NSFC: 41806215) and the "Key Laboratory of Intelligent Perception and Systems for High-Dimensional Information of Ministry of Education (Nanjing University of Science and Technology)" with the grant number 201804.

Acknowledgments: The radar and anemometer data were provided by the Institute of Oceanography, University of Hamburg.

Conflicts of Interest: The authors declare no conflicts of interest.

\section{References}

1. Crombie, D.D. Doppler spectrum of sea echo at 13.56 mcs. Nature 1955, 4459, 681-682. [CrossRef]

2. Long, A.E.; Trizna, D.B. Measurements and preliminary interpretation of hf radar Doppler spectra from the sea-echo of an atlantic storm. NRL Rep. 1972, 7456.

3. Stewart, R.H.; Barnum, J.R. Radio measurements of oceanic winds at long ranges: An evaluation. Radio Sci. 1975, 10, 853-857. [CrossRef]

4. Tyler, G.L.; Teague, C.C.; Stewart, R.H.; Peterson, A.M.; Munk, W.H.; Joy, J.W. Wave directional spectra from synthetic aperture observations of radio scatter. Deep Sea Res. Oceanogr. Abstr. 1974. [CrossRef]

5. Zeng, Y.; Zhou, H.; Lai, Y.; Wen, B. Wind-direction mapping with a modified wind spreading function by broad-beam high-frequency radar. IEEE Geosci. Remote Sens. Lett. 2018, 15, 679-683. [CrossRef]

6. Donelan, M.A.; Hamilton, J.; Hui, W.H. Directional spectra of wind-generated waves. Phil. Trans. R. Soc. Lond. 1985, 315, 509-562. [CrossRef]

7. Gurgel, K.W.; Essen, H.H.; Schlick, T. An empirical method to derive ocean waves from second-order Bragg scattering: Prospects and limitations. IEEE J. Ocean. Eng. 2006, 31, 804-811. [CrossRef]

8. Heron, M.L. Applying a unified directional wave spectrum to the remote sensing of wind wave directional spreading. Can. J. Remote Sens. 2002, 28, 346-353. [CrossRef]

9. Heron, M.L.; Prytz, A. Wave height and wind direction from the hf coastal ocean surface radar. Can. J. Remote Sens. 2002, 28, 385-393. [CrossRef]

10. Huang, W.; Gill, E.; Wu, S.; Wen, B.; Yang, Z.; Hou, J. Measuring surface wind direction by monostatic HF ground-wave radar at the eastern China sea. IEEE J. Ocean. Eng. 2004, 29, 1032-1037. [CrossRef]

11. Chu, X.; Zhang, J.; Ji, Y.; Wang, Y.; Yang, L. Extraction of wind direction from the hf hybrid sky-surface wave radar sea echoes. IEEE Aerosp. Electron. Syst. Mag. 2018, 33, 42-47. [CrossRef]

12. Shen, W.; Gurgel, K.W.; Voulgaris, G.; Schlick, T.; Stammer, D. Wind-speed inversion from HF radar first-order backscatter signal. Ocean. Dyn. 2012, 62, 105-121. [CrossRef]

13. Hisaki, Y. Sea Surface Wind Correction Using HF Ocean Radar and Its Impact on Coastal Wave Prediction. J. Atmos. Ocean. Technol. 2017, 34, 2001-2020. [CrossRef]

14. Kirincich, A. Toward real-time, remote observations of the coastal wind resource using high-frequency radar. Mar. Technol. Soc. J. 2013, 47, 206-217. [CrossRef]

15. Kirincich, A. Remote Sensing of the Surface Wind Field over the Coastal Ocean via Direct Calibration of HF Radar Backscatter Power. J. Atmos. Ocean. Technol. 2016, 33, 1377-1392. [CrossRef]

16. Zeng, Y.; Zhou, H.; Roarty, H.; Wen, B. Wind Speed Inversion in High Frequency Radar Based on Neural Network. Int. J. Antennas Propag. 2016, 2016. [CrossRef]

17. Li, X.; Yu, C.; Su, F.; Quan, T.; Chu, X.; Wang, S. Significant wave height extraction using a low-frequency HFSWR system under low sea state. IET Radar Sonar Navig. 2018, 12, 671-677. [CrossRef] 
18. Halverson, M.; Pawlowicz, R.; Chavanne, C. Dependence of 25-MHz HF Radar Working Range on Near-Surface Conductivity, Sea State, and Tides. J. Atmos. Ocean. Technol. 2017, 34, 447-462. [CrossRef]

19. Dexter, P.E.; Theodorides, S. Surface wind speed extraction from hf sky-wave radar doppler spectra. Radio Sci. 1982, 17, 643-652. [CrossRef]

20. Hasselmann, D.E.; Dunckel, M.; Ewing, J.A. Directional wave spectra observing during jonswap 1973. J. Phys. Oceanogr. 1980, 10, 1264-1280. [CrossRef]

21. Barrick, D.E. First-order theory and analysis of $\mathrm{mf} / \mathrm{hf} / \mathrm{vhf}$ surface from the sea. IEEE Trans. Atennas Propag. 1972, 20, 2-10. [CrossRef]

22. Robson, R.E. Simplified theory of first- and second-order scattering of hf radio waves from the sea. Radio Sci. 1984, 19, 1499-1504. [CrossRef]

23. Nagai, K. Diffraction of the irregular sea due to breakwaters. Coast. Eng. Japan. JSCE 1972, 15, 59-67. [CrossRef]

24. Borgman, L.E. Directional spectrum estimation for the sxy gauges. Tech. Rep. Coast. Eng. Res. Center Vicksburg 1984.

25. Longuet-Higgins, M.S.; Cartwright, D.E.; Smith, N.D. Observations of the directional spectrum of sea waves using the motions of a floating buoy. In Ocean Wave Spectra; Prentice-Hall Inc.: Upper Saddle River, NJ, USA, 1963; pp. 111-136.

26. Mitsuyasu, H.; Tasai, F.; Suhara, T.; Mizuno, S.; Ohkusu, M.; Honda, T.; Rikiishi, K. Observations of the directional spectrum of ocean waves using a cloverleaf buoy. J. Phys. Oceanogr. 1975, 5, 750-761. [CrossRef]

27. Chu, X.L.; Zhang, J.; Wang, S.Y.; Ji, Y.; et al. A Comparison of Algorithms for Extracting Wind Direction from the Monostatic HF Radar Sea Echoes. In Proceedings of the 2015 International Conference on Electronic Science and Automation Control, Zhengzhou, China, 15-16 August 2015.

(C) 2018 by the authors. Licensee MDPI, Basel, Switzerland. This article is an open access article distributed under the terms and conditions of the Creative Commons Attribution (CC BY) license (http:/ / creativecommons.org/licenses/by/4.0/). 